\title{
Booming Omics in Schistosoma
}

\author{
Le Govic Yohann ${ }^{1}$, Gourbal Benjamin 2, Boissier Jérôme 3, *
}

1 Department of Medical Parasitology and Mycology, Amiens University Hospital, Amiens, France 2 Host-Pathogen Interaction Study Group (GEIHP, EA 3142), UNIV Angers, UNIV Brest, Federative Structure of Research 'Cellular Interactions and Therapeutic Applications', SFR 4208 ICAT, Angers, France

${ }^{3}$ IHPE, University of Montpellier, CNRS, Ifremer, University of Perpignan Via Domitia, Perpignan, France

*Corresponding author : Jérôme Boissier, email address : boissier@univ-perp.fr

\begin{abstract}
:
Efforts to eliminate schistosomiasis are hindered by incomplete efficacy of the only FDA-approved antischistosomal drug, praziquantel. By using postgenomic technologies, Wendt et al. and Wang et al. deciphered the function of several genes required for worm survival and pathogenesis, which opens the way for the development of innovative parasite-targeted therapies.
\end{abstract}

Keywords : schistosomiasis, RNA interference, scRNA-seq, praziquantel proteasome, blood feeding 


\section{Manuscript}

Schistosomiasis - also known as bilharziasis - is one of the most devastating world's neglected tropical disease responsible for considerable human morbidity. The etiological agents, schistosomes, are dioecious flatworms that reside permanently in copula within the host blood vessels. While blood feeding, the females lay eggs of which the majority becomes encysted in surrounding host tissues and organs (especially the liver), causing pathology [1]. Despite increased efforts to eradicate schistosomiasis over the past five decades, more than 200000 people still die each year from schistosome-associated complications [2]. This apparent failure to control disease transmission is in part due to the incomplete parasiticidal efficacy of praziquantel, which is ineffective on immature worms, does not prevent reinfection or the emergence of drug-resistant parasites. Accordingly, in its recently published roadmap aiming at eliminating schistosomiasis by 2030, the World Health Organization (WHO) highlights that new anti-schistosomal treatments are urgently needed [3].

Hitherto, the development of innovative therapies has been hampered by the paucity of molecular and genetic tools for exploring schistosome biology. In their two enlightening articles, Wang et al. [4] and Wendt et al. [5] provide new insights into the functional biology of schistosomes that will undoubtedly unleash the discovery of new potential drug targets.

Indeed, despite tremendous advances in both genome resources and genetics in the past few years (Figure 1), much of the molecular and cellular processes that underpin the tissue maintenance and survival of adult schistosomes remain poorly 
understood. To gain a better insight into these processes, Wendt and colleagues joining FACS (Fluorescence-Activated Cell Sorting), single-cell transcriptomics and insitu hybridization successfully generate an unbiased classification of cellular subtypes in $S$. mansoni male, mature and immature female worms. This approach enabled to produce a precious atlas of $\sim 43,000$ high-quality cells (freely available at http://www.collinslab.org/schistocyte/), representing 68 transcriptionally distinct cell categories [5]. These clusters notably included neoblasts, which are motile stem cells involved in tissue turnover and regeneration [6]. Although previous studies indicate that neoblasts of schistosomes predominantly serve for tegument maintenance, there is evidence that other flatworms (e.g., Planarians) also possess 'specialized' neoblasts that are progenitors for specific differentiated tissues [7]. Here, Wendt et al. pointed out the existence of a previously undescribed sub-population of neoblasts that cluster with gut cells, the latter being required for digestion of host blood. By combining RNA interference (RNAi) and parasitological analyses, it was shown that this neoblast 'gut lineage' expresses a homologue of hepatocyte nuclear factor 4 (hnf4), a gut marker in planarians, which is required for gut preservation and hematophagous behavior. Indeed, RNA silencing of hnf4 resulted in a sizeable drop in the number of luminal microvilli, leading to morphological abnormalities of the gut and subsequent parasites' inability to digest red blood cells and absorb nutrients. Considering the importance of blood feeding for the production of eggs, which are the hallmarks of schistosomiasis pathology, Wendt et al. further evaluated the pathogenicity of worms whose hnf4 gene was knocked-down through RNAi. In a striking in vivo experiment, it was demonstrated that the loss of hnf4 confers reduced pathology (i.e., egginduced granulomas) following surgical transplantation into Schistosoma-naive mice. 
Moreover, RNAi-treated parasites were significantly shorter than non-treated controls, indicating that $h n f 4$ is also required for normal growth. Taken together, these data suggest that targeting the blood feeding machinery may constitute a relevant alternative strategy to praziquantel.

Another study conducted by the same research group has successfully used highthroughput RNAi screening for profiling the essential gene landscape, and the identification of novel therapeutic targets in $S$. mansoni [4]. By this approach, Wang and colleagues present a dataset of over 2000 genes ( 20\% of the protein-coding genes), of which 261 were assigned to the parasite's critical gene repertoire. From the list of top candidates, a couple of serine/threonine kinases (TAO and STK25) were found to be essential in maintaining neuromuscular function. Indeed, RNA silencing of either of these kinase encoding genes resulted in impaired locomotion, tetanic contraction of the body of the worm, and detachment from the culture substrate. Most importantly, loss of tao or stk25 gene expression impedes schistosome capacity to reside within the host blood vessels, resulting in an attenuated schistosome egg-induced pathology. Another noteworthy feature of this work is the elegant approach used to characterize a large portion of the 195 genes designated as essential for parasite attachment. Indeed, the transcriptomic analysis revealed substantial amounts of genes related to the ubiquitin-proteasome pathway (UPP), which maintains protein homeostasis and normal cellular functions in eukaryotes [8]. Strikingly, RNA silencing of virtually all UPP elements led to tissue degeneration in vitro, worm immobilization and even death [4]. This underscores a central role of UPP in the maintenance of tegument integrity and survival in schistosomes. Considering the importance of UPP in both larval and adult worm 
stages $[9,10]$, Wang et al. then sought to evaluate its druggability. By filtering databases and chemical libraries, a series of compounds were therefore selected upon their potential to inhibit UPP components. This last and most exciting approach allowed identifying p97, a central component in the UPP, as a novel target for drug development. In sum, this article sheds light on the usefulness of large-scale RNAi for unravelling complex cellular pathways and exploring their potential benefit in the field of schistosomiasis.

Altogether, these two illuminating articles provide unprecedented foundation for better understanding multiple aspects of schistosome biology and subsequently unveil hidden drug targets. These findings notably allowed putting in foreground some pre-existing pharmacological agents, thus accelerating the discovery of unexpected highly effective anthelminthic compounds. It is also important to underline the considerable value of the state-of-the-art methods employed here, in order to identify other clinical drug candidates with selective activity on worm (and thus with limited side-effects). One future investigation avenue may concern reproductive biology (i.e., egg production) of schistosomes, because such therapeutic intervention would combine the advantage of reducing the pathology while limiting the transmission. The latest exciting developments in functional genomic technologies (especially CRISPR-Cas9) will undoubtedly stimulate further fruitful research into this important area of schistosome biology and greatly help for disease control and development of innovative therapeutic strategies. 


\section{REFERENCES}

1 Colley, D.G. et al. (2014) Human schistosomiasis. The Lancet 383, 2253-2264

2 Chitsulo, L. et al. (2004) Schistosomiasis. Nat Rev Microbio/2, 12-13

3 Ending the neglect to attain the Sustainable Development Goals - A road map for neglected tropical diseases 2021-2030. World Health Organization: Geneva, 2020.

4 Wang, J. et al. (2020) Large-scale RNAi screening uncovers therapeutic targets in the parasite Schistosoma mansoni. Science 369, 1649-1653

5 Wendt, G. et al. (2020) A single-cell RNA-seq atlas of Schistosoma mansoni identifies a key regulator of blood feeding. Science 369, 1644-1649

6 Collins, J.J. et al. (2013) Adult somatic stem cells in the human parasite Schistosoma mansoni. Nature 494, 476-479

7 Reddien, P.W. (2018) The cellular and molecular basis for planarian regeneration. Cell 175, 327-345

8 Collins, G.A. and Goldberg, A.L. (2017) The logic of the 26S proteasome. Cell $169,792-806$

9 Bibo-Verdugo, B. et al. (2019) The proteasome as a drug target in the metazoan pathogen, Schistosoma mansoni. ACS Infect. Dis. 5, 1802-1812

10 Nabhan, J.F. et al. (2007) The 26S proteasome in Schistosoma mansoni: bioinformatics analysis, developmental expression, and RNA interference (RNAi) studies. Exp. Parasitol. 117, 337-347

Figure 1. Timeline of omics milestones and tendencies for Schistosoma research. 


\section{Functional genomics}

\section{High throughput screening}

2020

SINGLE-CELL RNA-SEQ

LARGE SCALE RNAI

EXOME SEQUENCING LNCRNAS

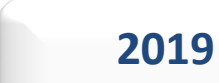

CRISPR-CAS9

\section{Computational drug repurposing}
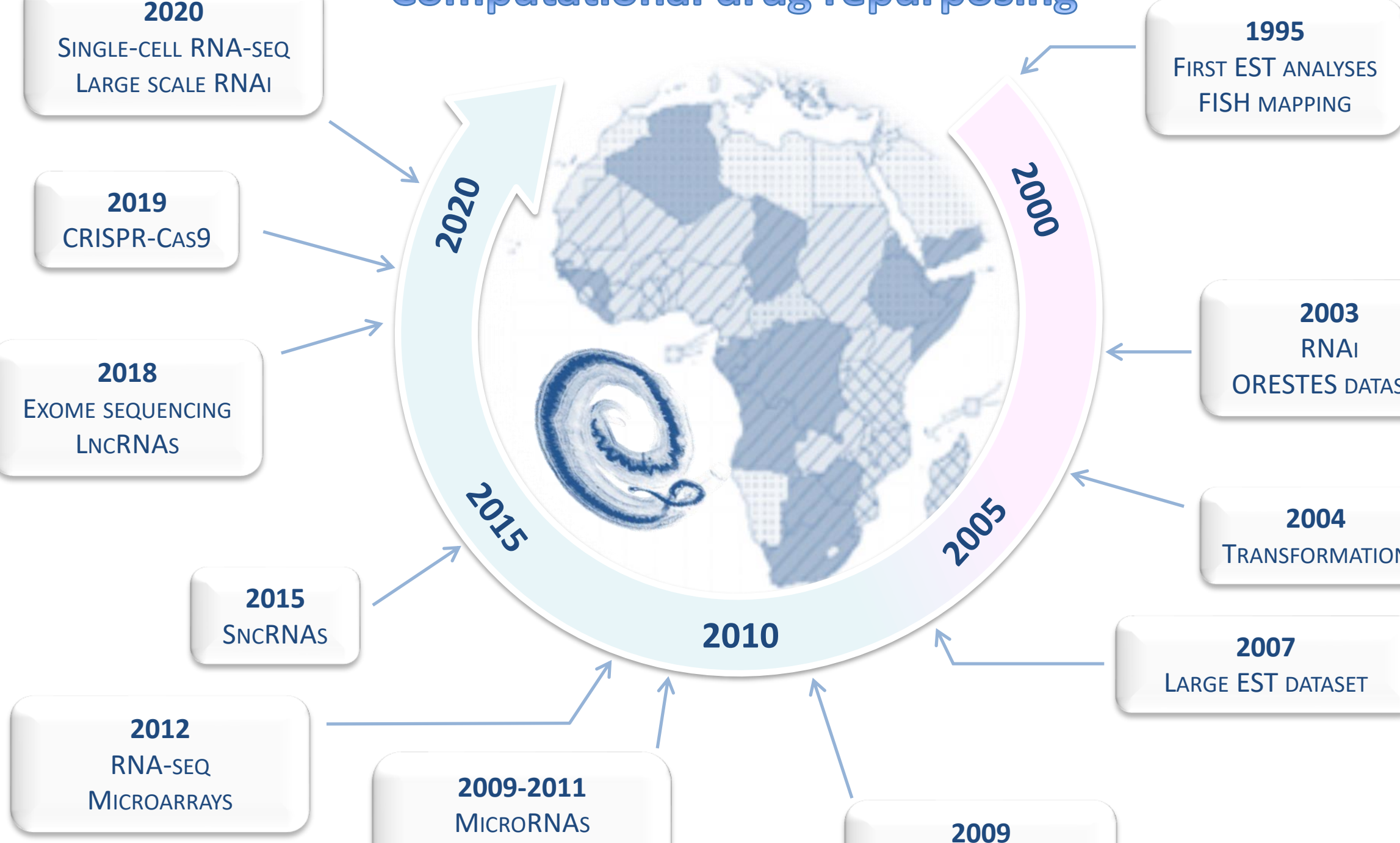

2009-2011

MICRORNAS

GENOME WIDE

COMPARISON 